J. Clin. Chem. Clin. Biochem.

Vol. 15, 1977, pp. 27-29

\title{
Der Einfluß von Pentagastrin auf den Gehalt an freien Aminosäuren im Magensaft
}

\author{
Von U. Dunzendorfer \\ Zentrum Chirurgie der Johann-Wolfgang von-Goethe-Universität Frankfurt/Main
}

und P. Beutelmann

Institut für Botanik, Johann-Gutenberg-Universität Mainz

(Eingegangen am 30. Juli 1975/10. August 1976)

Zusammenfassung: Die normo-, hyper- und anaciden Zustände der Magenschleimhaut unterscheiden sich signifikant in dem Gehalt an freien Aminosäuren ihres Magensaftes. Die freien Aminosäuren können im enteiweißten und entfetteten Magensaft säulenchromatographisch bestimmt werden. Die Konzentration der freien Aminosäuren nimmt 45 min nach Pentagastrinreiz gegenüber den Nüchternwerten außer bei Patienten mit Anacidität signifikant ab.

\section{The influence of pentagastrin on the concentration of free amino acids in the gastric juice}

Summary: The content of the free amino acids in the gastric juice is different in the state of normo-, hypo-, hyperand anacidity. Free amino acids can be determined by the amino autoanalyzer after deproteinisation and delipidation of the gastric juice. $45 \mathrm{~min}$ after pentagastrin stimulation the content of free amino acids is significantly reduced compared to the values before stimulation, except for patients with anacidity.

\section{Einleitung}

Pentagastrin besitzt die volle Gastrinaktivität und wird in der Routinediagnostik zur Differenzierung der säuresekretorischen Funktion der Magenschleimhaut verwendet (1). In der vorliegenden Arbeit soll geprift werden, ob Pentagastrin die freien Aminosäuren im Magensaft beeinflußt. Wir untersuchten säulenchromatogrạphisch den Magensaft von Patienten mit Normo-, Hypo-, Hypèr- und Anacidität auf den Gehalt an freien Aminosäuren. Als Bezugsparameter wurden die Volumina und HCl-Produktion gemessen und versucht, Körrelationen zwischen diesen und der Freisetzungsrate der Aminosäuren zu finden.

\section{Methodik}

Die Magensäfte wự̣̂den im Rahmen des Pentagastrintestes, durchgefuhrt in typischer Art (2), gewonnen. Die Probanden wurden in vier Gruppen eingeteillt. Dàs Kontrollkollektiv ( $N=30,18-50$ Jahre) mit einer Säuremenge bis zu $40 \mathrm{mmol} / \mathrm{h}$ ñach Pentagastrinreiz, die Gruppe mit Hypoacidität $(\mathrm{N}=10,30-60 \mathrm{Jahre}) \mathrm{mit}$ einer Säuremenge bis zu $25 \mathrm{mmol} / \mathrm{h}$, die Gruppe mit Hyperacidität $(\mathrm{N}=10,22-50 \mathrm{Jahre})$ mit einer Säuremenge über $40 \mathrm{mmol} / \mathrm{h}$ und die Gruppe mit Anacidität ( $N=10,45-71$ Jahre, ohne carcinomatöse Begleiterkrankungen) mit ẹner Säuremenge unter
$2 \mathrm{mmol} / \mathrm{h}$. Die Diagnose chronisch-atrophischer Gastritis wurde aus der Biopsie der Corpusschleimhaut gestellt. Sofort nach dem Auftauen wurden die Proben mit Aceton enteiweißt und der Uberstand im Rotationsverdampfer bei $60^{\circ} \mathrm{C}$ getrocknet. Der in bidest. Wasser gelöste Rückstand wurde mit gleichen Mengen Petroläther $\left(\mathrm{Kp} 60-80^{\circ} \mathrm{C}\right)$ im Scheidetrichter getrennt, die wäßrige Phase mit $1 \mathrm{~mol} / 1 \mathrm{HCl}$ auf $\mathrm{pH} 2$ angesäuert und jeweils $1 \mathrm{ml}$ pro Säule auf den Aminosäureanalysator Unichrom aufgetragen. Die Aminosäureanalyse erfolgte nach der Methode von Spackman (3) unter Verwendung der yon dem Hersteller angegebenen Puffer. Die Auswertung erfolgte anhand von Standardchromatogrammen und nur eindeutig identifizierbare Aminosäuren wurden in die statistische Berechnung (gepaarter und ungepaarter t-Test) aufgenommen. Daneben erschienen auf den Chromatogrammen der sauren Aminosäuren nach 35, 40, 48, 165, 175, 182,240 und 285 min noch weitere nicht identifizierbare Peaks, auf dem der basisischen Aminosäuren fand sich nach $190 \mathrm{~min}$ ein noch nicht identifizierter Peak. Tryptophan ließ sich mit unserer Versuchsanordnung nicht sicher trennen.

\section{Ergebnisse}

Der Aminosäuregehalt des Nüchternsekretes bei Patienten mit Anacidität ist mit 526,3 $\pm 349,2 \mathrm{mmol} / 15 \mathrm{~min}$ (p $<0,05)$ deutlich erhöht, bei denen mit Hyperacidität $(65,4 \pm 18,2 \mathrm{mmol} / 15 \mathrm{~min}, \mathrm{p}=0,001)$ signifikant erniedrigt, bei denen mit Hypoacidität $(152 \pm 88,5 \mathrm{mmol} / \mathrm{l})$ 
errechneten sich keine Unterschiede zu dem Kontrollkollektiv (146.4 $\pm 72,2 \mathrm{mmol} / 15 \mathrm{~min}) ; 15 \mathrm{~min}$ nach Pentagastrinreiz ist der Aminosäuregehalt bei Patienten mit Hyperacidität $(54.7 \pm 20.7 \mathrm{mmol} / 15 \mathrm{~min}$, $p=0,0001$ ) signifikant zu dem des Nüchternwertes erniedrigt, bei den übrigen Kollektiven ergeben sich keine Differenzen.

45 min nach Pentagastrinreiz ist der Aminosäuregehalt bei allen Untersuchungsgruppen (Normacidität:

$102,3 \pm 61,2 \mathrm{mmol} / 15 \mathrm{~min}, \mathrm{p}<0,05$, Hypoacidität: $60,6 \pm 19,0 \mathrm{mmol} / 15 \mathrm{~min}, \mathrm{p}<0,05$, Hyperacidität: $24,2 \pm 7,2 \mathrm{mmol} / 15 \mathrm{~min}, \mathrm{p}=0,0001$ ) deutlich gegenüber den Ausgangswerten außer bei Patienten mit Anacidität (1041,0 $\pm 795,8 \mathrm{mmol} / 15 \mathrm{~min})$ gesunken.

\section{Einzelne Aminosäuren}

15 min nach Pentagastrinreiz sind bei Patienten mit Normacidität Lys, Asp, Ala, Cys (1/2), Asn, Cịt, Phe, Pro signifikant verändert, 45 min später sind alle Aminosäuren gegenüber den Nüchternwerten um den Faktor $0,1-0,05$ signifikant erniedrigt $(p<0,01)$ (Tab. 1).

Bei Patienten mit Hypoacidität ist 15 min nach Reiz Met signifikant erhöht, 45 min später sind Asp, Glu, Ala, Cys, Gly, Ser, Val, Asn, Leu, Orn, Phe, Pro, Tyr um den Faktor 0,01-0,025 gegenüber den Nüchternwerten signifikant erniedrigt ( $p<0,5>0,01$ ) (Tab. 1).
Bei Patienten mit Hyperacidität sind 15 min nach Reiz bis auf Arg (unverändert) und Asp (deutlich erhöht) alle übrigen Aminosäuren signifikant ( $p<0,05>0,001)$ erniedrigt, 45 min später sind alle Aminosäuren um den Faktor $0,2-0,8$ signifikant gegenüber den Nüchternwerten verringert $(p<0,001)$ (Tab. 1).

Bei Patienten mit Anacidität sind 15 min nach Reiz Arg und Ile erhöht, 45 min später liegen dieselben Ergebnisse vor (Tab. 1).

\section{Diskussion}

Die freien Aminosäuren im Magensaft nehmen nach Injektion von Pentagastrin bis auf das Untersuchungskollektiv der Patienten mit Anacidität ab. Alle gemessenen Konzentrationen vor und nach Pentagastrinreiz liegen weit unter den Plasmawerten. Pentagastrin steigert die Volumen- und $\mathrm{HCl}-S e k r e t i o n$ in der Magenschleimhaut (1, 2), der Eiweißgehalt im Magensaft bleibt unverändert $(4,5)$ und der Aminosäuregehalt nimmt ab. Bei Patienten mit Hyperacidität ist die Konzentration an freien Aminosäuren um $50 \%$ gegenüber der Norm verringert und erklärt sich mit der auf das Doppelte gesteigerten Volumensekretion. Die hohen Konzentrationen an freien Aminosäuren bei Patienten mit Anacidität entstehen durch Zellnekrosen der chronisch-atrophisch entzündeten Magenschleimhaut und intrazellulär akkumulierte Amino-

Tab. 1. Darstellung der Konzentrationen der freien Aminosäuren im Magensaft während einer 15 min Sammelperiode vor Pentagastrinreiz bei den vier verschiedenen säuresekretorischen Funktionszuständen der Magenschleimhaut. Die Signifikanz wurde im ungepaarten t-Test mit zweiseitiger Fragestellung geprüft. Für Asparaginsäure und Glutaminsäure muß es in der Gruppe der Anacidität heißen: Aspartat und Glutamat.

\begin{tabular}{|c|c|c|c|c|c|c|c|c|c|c|c|}
\hline \multirow[b]{2}{*}{$\begin{array}{l}\text { Arginin } \\
\text { Lysin }\end{array}$} & \multicolumn{2}{|c|}{$\begin{array}{l}\frac{\text { Normacide }}{\mathrm{x}} \mathrm{s} \\
\mathrm{mmol} / 15 \mathrm{~min}\end{array}$} & \multicolumn{2}{|c|}{$\begin{array}{l}\text { Hypoacide } \\
\bar{x} \quad s \\
\text { mmol/15 min }\end{array}$} & \multirow{2}{*}{$\begin{array}{l}P \\
0,01 \\
0,05\end{array}$} & \multicolumn{2}{|c|}{$\begin{array}{l}\text { Hyperacide } \\
\bar{x} \\
\text { mmol/15 } \mathrm{min}\end{array}$} & \multirow{2}{*}{$\begin{array}{l}P \\
0,005 \\
0,05\end{array}$} & \multicolumn{2}{|c|}{$\begin{array}{l}\bar{x}_{\mathrm{x}} \mathrm{s} \\
\mathrm{mmol} / 15 \mathrm{~min}\end{array}$} & \multirow{2}{*}{$\begin{array}{l}P \\
0,001 \\
0,1\end{array}$} \\
\hline & $\begin{array}{l}33,9 \\
11,8\end{array}$ & $\begin{array}{r}17,8 \\
7,0\end{array}$ & $\begin{array}{l}6,15 \\
6,45\end{array}$ & $\begin{array}{l}2,45 \\
2,63\end{array}$ & & $\begin{array}{l}5,17 \\
8,15\end{array}$ & $\begin{array}{l}0,75 \\
1,13\end{array}$ & & $\begin{array}{r}9,51 \\
25,94\end{array}$ & $\begin{array}{r}7,13 \\
21,06\end{array}$ & \\
\hline $\begin{array}{l}\text { Asparaginsäure } \\
\text { Glutaminsäure }\end{array}$ & $\begin{array}{l}4,0 \\
4,3\end{array}$ & $\begin{array}{l}2,2 \\
2,7\end{array}$ & $\begin{array}{l}3,13 \\
5,40\end{array}$ & $\begin{array}{l}1,28 \\
2,20\end{array}$ & $\begin{array}{l}0,3 \\
0,5\end{array}$ & $\begin{array}{l}0,68 \\
2,22\end{array}$ & $\begin{array}{l}0,09 \\
0,31\end{array}$ & $\begin{array}{l}0,001 \\
0,002\end{array}$ & $\begin{array}{l}18,55 \\
14,96\end{array}$ & $\begin{array}{l}15,02 \\
12,11\end{array}$ & $\begin{array}{l}0,05 \\
0,05\end{array}$ \\
\hline $\begin{array}{l}\text { Alanin } \\
1 / 2 \text { Cystin } \\
\text { Glycin } \\
\text { Serin } \\
\text { Valin }\end{array}$ & $\begin{array}{c}14,3 \\
0,15 \\
7,9 \\
8,5 \\
9,3\end{array}$ & $\begin{array}{r}11,1 \\
0,1 \\
4,3 \\
4,9 \\
4,9\end{array}$ & $\begin{array}{c}12,43 \\
1,65 \\
4,8 \\
8,4 \\
9,15\end{array}$ & $\begin{array}{l}5,08 \\
0,67 \\
1,95 \\
3,42 \\
3,73\end{array}$ & $\begin{array}{l}0,5 \\
0,01 \\
0,005 \\
0,8 \\
0,8\end{array}$ & $\begin{array}{l}3,77 \\
1,80 \\
1,85 \\
1,67 \\
2,56\end{array}$ & $\begin{array}{l}0,51 \\
0,31 \\
0,29 \\
0,22 \\
0,36\end{array}$ & $\begin{array}{l}0,005 \\
0,001 \\
0,001 \\
0,001 \\
0,001\end{array}$ & $\begin{array}{r}28,84 \\
2,98 \\
21,06 \\
19,92 \\
21,46\end{array}$ & $\begin{array}{r}27,30 \\
2,43 \\
17,08 \\
16,18 \\
17,42\end{array}$ & $\begin{array}{l}0,5 \\
0,01 \\
0,05 \\
0,1 \\
0,1\end{array}$ \\
\hline $\begin{array}{l}\text { Asparagin } \\
\text { Citrullin } \\
\text { Histidin } \\
\text { Isoleucin } \\
\text { Leucin } \\
\text { Methionin } \\
\text { Ornithin } \\
\text { Phenylalanin } \\
\text { Prolin } \\
\text { Threonin } \\
\text { Tyrosin } \\
\end{array}$ & $\begin{array}{r}9,0 \\
0,7 \\
1,9 \\
6,5 \\
18,9 \\
6,0 \\
0,0 \\
22,1 \\
7,2 \\
6,3 \\
12,1 \\
\end{array}$ & $\begin{array}{r}5,2 \\
0,5 \\
1,1 \\
4,6 \\
12,9 \\
4,3\end{array}$ & $\begin{array}{r}5,7 \\
0,0 \\
1,6 \\
5,9 \\
17,8 \\
3,6 \\
0,4 \\
11,4 \\
3,4 \\
7,8 \\
8,2 \\
\end{array}$ & $\begin{array}{l}2,32 \\
0,0 \\
0,6 \\
2,6 \\
7,28 \\
1,46 \\
0,1 \\
4,6 \\
1,4 \\
3,1 \\
3,3\end{array}$ & $\begin{array}{l}0,05 \\
0,8 \\
0,5 \\
0,5 \\
0,8 \\
0,05\end{array}$ & $\begin{array}{l}1,29 \\
0,2 \\
1,24 \\
1,3 \\
9,0 \\
1,15 \\
0,0 \\
6,8 \\
1,85 \\
1,41 \\
3,7\end{array}$ & $\begin{array}{l}0,18 \\
0,03 \\
0,67 \\
0,15 \\
1,37 \\
0,27\end{array}$ & $\begin{array}{l}0,001 \\
0,001 \\
0,8 \\
0,001 \\
0,05 \\
0,001\end{array}$ & $\begin{array}{c}12,34 \\
3,11 \\
8,0 \\
1,08 \\
50,0 \\
5,0 \\
2,75 \\
14,9 \\
34,3 \\
15,8 \\
1,0\end{array}$ & $\begin{array}{c}10,05 \\
2,54 \\
6,51 \\
0,88 \\
36,27 \\
4,1 \\
1,98 \\
12,1 \\
27,7 \\
12,8 \\
0,8 \\
\end{array}$ & $\begin{array}{l}0,5 \\
0,05 \\
0,05 \\
0,05 \\
0,05 \\
0,5\end{array}$ \\
\hline
\end{tabular}


säuren werden dabei freigesetzt. Die unterschiedlich ausgeprägten Formen der chronisch-atrophischen Gastritis füren zu den hohen Standardabweichungen. Zusammenfassend läßt sich sagen, daß Pentagastrin bei Patienten mit Norm- und Hyperacidität die Sekretion der freien

\section{Literatur}

1. Brooméa, N., Fyrö, E. \& Olbe, L. (1968), Acta Physiol. Scand. 74, 331-339.

2. Ottenjann, R., Link, H. \& Hadelmann, O. (1968), Dtsch. Med. Wochenschr. 93, 2269-2271.

3. Spackman, D. H., Stein,W. H. \& Moore, S. (1954), Anal. Chem. 30, 1190-1206.
Aminosäuren nicht beeinflußt, bei Patienten mit Hypoacidität wird Sekretion einiger weniger Aminosäuren in den Magensaft gesteigert und bei Patienten mit Anacidität wird die Sekretion aller freien Aminosäuren in den Magensaft gesteigert.
4. Johnson, L. R., Aures, D. \& Yuen, L. (1969), Am. J. Physiol. 1217, 251-259.

5. Chandler, A. M. \& Johnson, L. R. (1972), Soc. Exp. Biol. Med. $141,110-113$.

Dr. Udo Dunzendorfer

Zentrum Chirurgie der

Johann-Wolfgang von-Goethe-Universität

D-6000 Frankfurt/Main 
